\section{A fluorescence lifetime extraction algorithm based on multiple signal classification}

\section{Hongqi Yu and David Day-Uei Li}

The multiple signal classification algorithm has been widely used in array signal processing for direction-of-arrival estimations. In this paper, we applied this algorithm to estimate fluorescence lifetimes for fluorescence lifetime imaging microscopy (FLIM) for the first time to our knowledge. Monte Carlo simulations were carried out to test its performances in comparison with the previously reported integral equation method, center-of-mass method and phasor method. Simulation results show that the proposed algorithm can achieve comparable or better results than the others. More importantly, it can easily resolve multi-exponential decays.

Introduction: When a fluorescent molecule is excited to the excited state by a pulsed laser, it can emit photons through fluorescing and return to the ground state. The fluorescence lifetime is the average time that the molecule stays in the excited state, and it is defined as the time delay for the fluorescence intensity to decrease to $1 / e$ of its original intensity. Fluorescence lifetime imaging microscopy (FLIM) is a powerful tool for research in biology, material sciences, chemical analysis, etc. It can be also used to reveal protein-protein interaction networks in living cells.

There are time-domain and frequency-domain FLIM instruments. Frequency-domain instruments usually use a modulated light source, and the phase difference between the modulated light and the fluorescence is calculated to obtain fluorescence lifetimes. Timedomain FLIM methods usually use a pulsed laser to excite the samples and use gated cameras or time-correlated single photo-counting (TCSPC) systems [1] to obtain lifetimes. TCSPCs are gold standard instruments widely used for FLIM applications. In a TCSPC module, a histogram of time delays between the detected photons and the pulsed laser is built for lifetime extractions. Traditional FLIM software are usually based on iterative algorithms such as maximum likelihood or least square methods, but they are computationally intensive and slow [2]. Non-iterative methods have been developed to solve such a problem. Leray et al. [3] used the phasor approach, whereas Li et al. [4] proposed a simple integral equation method (IEM) and later the centreof-mass method (CMM) [5].

In this paper, we proposed a new FLIM analysis method based on the multiple signal classification method. It was first proposed by Schmidt in 1986 for locating multiple emitters and estimating radio signal parameters [6]. Due to its effectiveness, it has been widely used in array signal processing for direction-of-arrival (DOA) estimations. This signal classification method can be also effective for FLIM analysis.

Theory: Suppose the number of the lifetime components is $P$. Following the model proposed by Hall and Selinger [7], the fluorescence intensity can be expressed as

$$
y(t)=\sum_{j=1}^{P}\left(f_{D j} e^{-t / \tau_{j}}\right)+n(t),
$$

where $f_{D j}$ is the fraction of the $j$-th component, $\tau_{j}$ is the lifetime of the $j$ th component [7], and $n(t)$ is the additive shot noise.

The photo count in the time bin $m h$ (h is the resolution of the TCSPC) is

$$
y(m h)=\sum_{j=1}^{P}\left(f_{D j} e^{-m h / \tau_{j}}\right)+n(m h) .
$$

In order to apply the multiple signal classification method into the lifetime estimations, we can arrange the counts from all time bins in the histogram as

$$
\mathrm{Y}=\left(\begin{array}{cccc}
1 & 1 & \cdots & 1 \\
e^{-\frac{h}{\tau_{1}}} & e^{-\frac{h}{\tau_{2}}} & \cdots & e^{-\frac{h}{\tau_{P}}} \\
\vdots & \vdots & \ddots & \vdots \\
e^{-\frac{(M-1) h}{\tau_{1}}} & e^{-\frac{(M-1) h}{\tau_{2}}} & \cdots & e^{-\frac{(M-1) h}{\tau_{P}}}
\end{array}\right)\left(\begin{array}{c}
f_{D 1} \\
f_{D 2} \\
\vdots \\
f_{D P}
\end{array}\right)+\left(\begin{array}{c}
n(0) \\
n(h) \\
\vdots \\
n((M-1) h)
\end{array}\right)
$$

$=A S+N$, where $M$ is the number of the time bins in the histogram.

Then we can obtain the covariance matrix of the output as
$R_{I}=E\left[Y Y^{H}\right]=E\left[(A S+N)(A S+N)^{H}\right]=A R_{S} A^{H}+\sigma^{2} \Sigma_{0}$,

where $(\cdot)^{H}$ represents the Hermitian transpose of the matrix and $\Sigma_{0}$ is the correlation matrix of the noise.

Here we apply a theorem: Let $\lambda_{i}$ and $u_{i}, i=1,2, \cdots, M$, be the solutions for $R_{x} u=\lambda \Sigma_{0} u$, where $\lambda_{i}$ are listed in the descending order. Then $\lambda_{i}$ and $u_{i}$ are the eigenvalues and corresponding eigenvectors of the matrix pencil $\left(R_{x}, \Sigma_{0}\right)$. If $R_{S}$ is a full rank matrix, then each column of $A$ is orthogonal to the matrix $U_{n}=\left[\begin{array}{llll}u_{P+1} & u_{P+2} & \cdots & u_{M}\end{array}\right]$. The proof can be found in the Ref [6]. When we obtain the noise subspace $U_{n}$, let

$$
Q_{\text {search }}(\tau)=1 /\left\|U_{n}^{H} A(\tau)\right\|^{2},
$$

where $A(\tau)=\left|\begin{array}{llll}1 & e^{h / \tau} & \cdots & e^{(M-1) h / \tau}\end{array}\right|$.

The $P$ largest peaks found in $Q_{\text {search }}(\tau)$ are corresponding to $\tau_{j}, j=$ $1, \ldots, P$. If we use the data from all the time bins directly, the computation is more complex and the performance will be poorer. To reduce the effects caused by the shot noise, we can firstly obtain a new histogram by merging several bins into a wider bin. A histogram with a lower number of bins can greatly reduce the requirement for data storage in the TCSPC system. Secondly, Eq. (1) is used to obtain the covariance matrix. After carrying out the singular value decomposition (SVD) on the covariance matrix, we use Eq. (2) to obtain the spectrum of a fluorescence lifetime distribution. By searching the largest $P$ peaks, we can locate the fluorescence lifetimes.

Fig. 1(a) shows a histogram to be analysed, where $M=1024$ and the photons in the first bin $N_{l}=1000$. Fig. 1(b) shows a new histogram ( $M$ $=15$ ) merged from Fig. 1(a), and it is smoother than the original one.
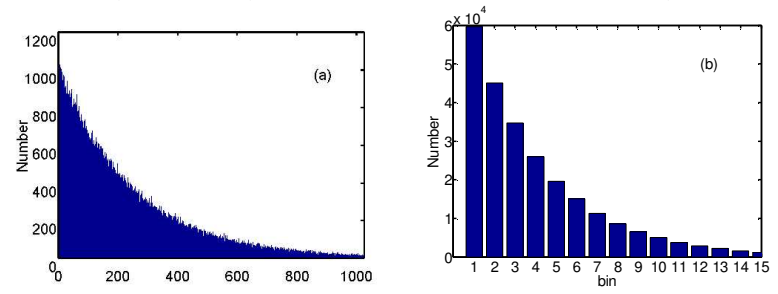

Fig.1 (a) Original histogram and (b) merged histogram.

Suppose the repetition rate of the laser is $80 \mathrm{MHz}$; the width of the measurement window $T=12.5 \mathrm{~ns}$. For this simulation, we consider a biexponential decay $(P=2)$ with $N_{l}=10000$ and $M=1024$ (the histogram was merged into a new one with $M=15$ ). As the signals are correlated, we can use the forward smoothing technique to obtain the new covariance matrix [8]. The proposed method, denoted as the MSC hereafter, is shown in Fig. 2. In this simulation, $f_{D 2}=1-f_{D 1}$. Figure 2 shows different spectra with different combinations, but they all have two peaks showing bi-exponential decays. For $\tau_{1}=1.6 \mathrm{~ns}, \tau_{2}=3.0 \mathrm{~ns}$ and $f_{D I}=0.2$, the spectrum estimates that $\tau_{1}=1.62 \mathrm{~ns}$ and $\tau_{2}=2.95 \mathrm{~ns}$.

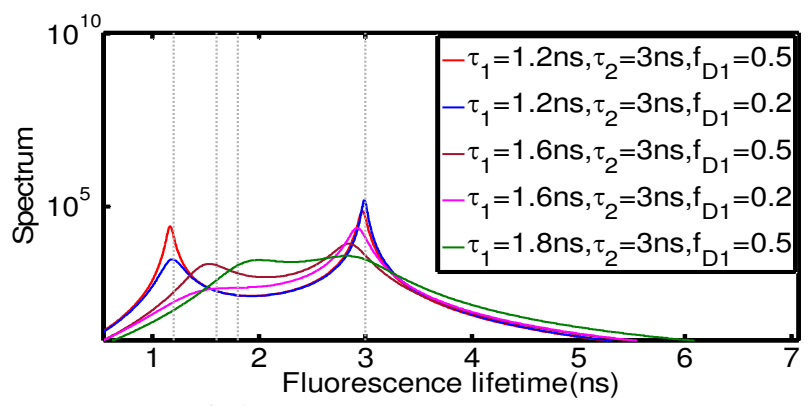

Fig.2 Spectrum of the MSC method.

Monte-Carlo Simulations: To demonstrate the bias and precision performances of the MSC, we define $\Delta \tau=\tau-\tau_{\mathrm{AVE}}$ and $F=N_{C}{ }^{0.5} \sigma \tau / \tau_{\mathrm{AVE}}$ [9], where $N_{C}$ is the number of all photons in the histogram and $\tau_{\mathrm{AVE}}$ is the mean of the estimation.

The bias performances are shown in Fig. 3. Here a single-exponential decay $\left(f_{D I}=1.0\right)$ is used in order to compare with other algorithms. For IEM and MSC, $M=15$. For CMM method and phasor method, $M=$ 1024. In Fig. 3(a), $\tau_{1}=3.0$ ns. In Fig.3 (b), we fixed $N_{l}=1000$ by 
varying $T / \tau$ with the other parameters being the same with those of Fig. 3(a). The $F$-value plots for different methods are shown in Fig.4.
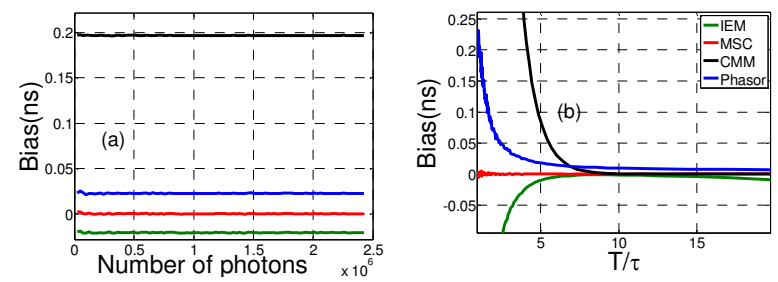

Fig.3 Comparisons of the bias performances.

The MSC has the best performance in bias, and the simulation results show that the $F$-value is about 1.7 . The $F$ value of the CMM is slightly lower than 1 , and that is because the CMM has a small bias.

Conclusion: In this paper, we applied the multiple signal classification method for the first time to analyse FLIM data and coined it as MSC. Simulations show that the MSC is the least biased among four methods. The $F$-value of the new method is also rather good. More importantly, the MSC is able to analyse blind FLIM analysis where the number of exponents are unknown.
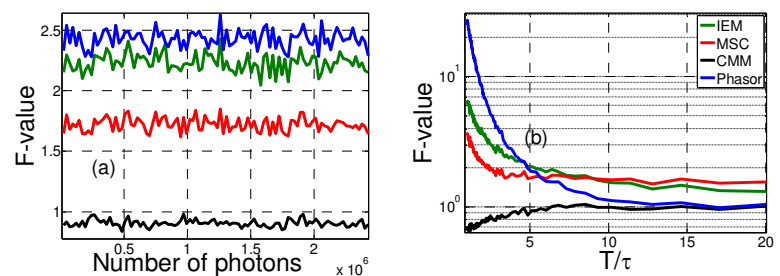

Fig.4 $F$-value for different methods.

\section{Acknowledgments}

This work was supported by the China Scholarship Council, China.

Hongqi Yu, School of Electronic Science and Engineering, National University of Defense Technology, Changsha, China, E-mail: 13755132901@163.com

David Day-Uei Li, Centre for Biophotonics, Strathclyde Institute of Pharmacy \& Biomedical Sciences, University of Strathclyde, Glasgow, UK.

\section{References}

1. Turgeman, L. and D. Fixler, Photon Efficiency Optimization in Time Correlated Single Photon Counting Technique for Fluorescence Lifetime Imaging Systems. IEEE Trans. Biomed. Eng., 2013. 60(6): p. 1571-1579.

2. Maus, M., et al., An Experimental Comparison of the Maximum Likelihood Estimation and Nonlinear Least-Squares Fluorescence Lifetime Analysis of Single Molecules. Anal. Chem., 2001. 73(9): p. 2078-2086.

3. Leray, A., et al., Generalization of the polar representation in time domain fluorescence lifetime imaging microscopy for biological applications: practical implementation. J. Microsc., 2012. 248(1): p. 66-76.

4. Li, D.-U., et al., On-Chip Time-Correlated Fluorescence Lifetime Extraction Algorithms and Error Analysis. J. Opt. Soc. Am. A, 2008. 25(5): p. 1190-1198.

5. Li, D.D.U., et al., Video-rate fluorescence lifetime imaging camera with CMOS single-photon avalanche diode arrays and high-speed imaging algorithm. J. Biomed. Opt., 2011. 16(9): p. 096012.

6. Schmidt, R.O., Multiple emitter location and signal parameter estimation. IEEE Trans. Antennas Propag., 1986. 34(3): p. 276-280.

7. Hall, P. and B. Selinger, Better estimates of exponential decay parameters. The Journal of Physical Chemistry, 1981. 85(20): p. 2941-2946.

8. Williams, R.T., et al., An improved spatial smoothing technique for bearing estimation in a multipath environment. Acoustics, Speech and Signal Processing, IEEE Transactions on, 1988. 36(4): p. 425-432.

9. Gerritsen, H.C., et al., Fluorescence lifetime imaging in scanning microscopes: acquisition speed, photon economy and lifetime resolution. Journal of Microscopy, 2002. 206(3): p. 218-224. 